PROCEEDINGS OF THE

AMERICAN MATHEMATICAL SOCIETY

Volume 42, Number 1, January 1974

\title{
ON TORSION ABELIAN GROUPS QUASI-PROJECTIVE OVER THEIR ENDOMORPHISM RINGS
}

\author{
LASZLO FUCHS ${ }^{1}$
}

\begin{abstract}
It is shown that a torsion abelian group is quasiprojective over its endomorphism ring exactly if, for every prime $p$, its $p$-component is bounded or has an unbounded basic subgroup.
\end{abstract}

Recently, a number of papers have dealt with the behavior of abelian groups $A$ regarded as modules over their endomorphism rings $E(A)=E$. For instance, Richman and Walker [3] have shown that an abelian $p$ group is flat as an $E$-module if and only if it is either bounded or has an unbounded basic subgroup. In another paper [4], they described all abelian groups which are injective as modules over their endomorphism rings. Poole and Reid [1] raised the question of abelian groups quasiinjective over their endomorphism rings; they have shown that all unmixed divisible groups and direct sums of finite cyclic groups share this property. Subsequently, Richman [2] proved that the class of p-groups, quasi-injective over their respective endomorphism rings, is fairly large: it includes all $p$-groups without elements of infinite height, the totally projective $p$-groups and all nonreduced $p$-groups.

In this note, we wish to raise the dual question: Which abelian groups are quasi-projective over their endomorphism rings? This question can be fully answered for torsion groups by the following simple result.

THEOREM. ${ }^{2} A$ torsion abelian group is quasi-projective as a module over the ring of its endomorphisms if and only if, for every prime $p$, its p-component is either bounded or has an unbounded basic subgroup.

It is a routine exercise to show that it suffices to consider $p$-groups $A$.

Suppose that $A$ is a $p$-group as stated, $G$ is an $E$-submodule of $A$ (i.e. a fully invariant subgroup of $A$ ) and $\phi: A \rightarrow A / G$ is the natural homomorphism. What we have to prove is that for every $E$-homomorphism $\alpha: A \rightarrow A / G$,

Received by the editors January 19, 1973.

AMS (MOS) subject classifications (1970). Primary 20K10, 16A50.

Key words and phrases. Abelian torsion group, endomorphism ring, quasi-projective module.

1 This paper was written while the author held a National Science Foundation grant, GP-29437.

${ }^{2}$ This result immediately generalizes to torsion modules over Dedekind domains.

(C) American Mathematical Society 1974 
there exists an endomorphism $\lambda$ of $A$ making the diagram

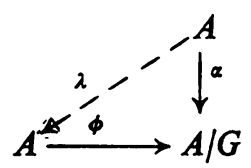

commutative. The hypothesis of $\alpha$ being an $E$-map is equivalent to saying that, for every $\eta \in E$,

$$
\bar{\eta} \alpha=\alpha \eta
$$

where $\bar{\eta}$ denotes the map $A / G \rightarrow A / G$ induced by $\eta$, i.e. $\bar{\eta}(a+G)=\eta a+G$ for $a \in A$.

Let $\langle a\rangle$ be a summand, of order $p^{n}$, of $A$, and write $A=\langle a\rangle \oplus C$. Then the full invariance of $G$ implies $G=(\langle a\rangle \cap G) \oplus(C \cap G)$, and hence we get a direct decomposition

$$
A / G=(\langle a\rangle+G) / G \oplus(C+G) / G .
$$

Let $\eta$ be the endomorphism of $A$ which is multiplication by an integer $t$ on $C$ and which satisfies $\eta a=a+x$ where $x$ is some element in $C\left[p^{n}\right]$. We can write

$$
\alpha a=k \bar{a}+\bar{c}
$$

where bars indicate cosets $\bmod G, c \in C$ and $k$ is an integer. Since $\bar{\eta} \bar{a}=$ $\bar{a}+\bar{x}, \bar{\eta} \bar{c}=t \bar{c}$, from (1) we deduce that

$$
\begin{aligned}
& \bar{\eta} \alpha a=\bar{\eta}(k \bar{a}+\bar{c})=k \bar{a}+k \bar{x}+t \bar{c}, \\
& \alpha \eta a=\alpha(a+x)=k \bar{a}+\bar{c}+\alpha x
\end{aligned}
$$

are equal, for every $t$. Therefore $\bar{c}=0, \alpha a=k \bar{a}$ and

$$
\propto x=k \bar{x} \text { for all } x \in A\left[p^{n}\right] \text {. }
$$

Suppose that $A$ has a summand $\langle b\rangle$ of order $>p^{n}$. Then we get similarly $\alpha x=k^{\prime} \bar{x}$ for some integer $k^{\prime}$ and for all $x \in A[o(b)]$, thus $k \equiv k^{\prime} \bmod o(\bar{a})$. It is now easy to conclude that if $A$ has an unbounded basic subgroup, then there is a $p$-adic integer $\pi$ such that $\alpha x=\pi \bar{x}$ for all $x \in A$. Consequently, if we choose $\lambda$ in the diagram to be the multiplication by this $\pi$, then the arising triangle will commute.

If $A$ is a bounded p-group and if $\langle a\rangle$ is a cyclic summand of maximal order, then (2) shows that $\lambda$ can be chosen as a multiplication by $k$. (Bounded $p$-groups are actually projective as $E$-modules; see Richman and Walker [3].)

In the remaining case, $A$ is of the form $A=B \oplus D$ where $B$ is bounded (say, $p^{m-1} B=0$ ) and $D$ is divisible $\neq 0$. Let $G=A\left[p^{m}\right]$ and select an $E$ homomorphism $\alpha: A \rightarrow A / G$ such that $\alpha B=0$ and $\alpha \mid D$ is monic. This 
can be done, for instance, by first writing $D=\bigoplus_{i \in I} D_{i}$ with $D_{i}=$ $\left\langle c_{i 1}, \cdots, c_{i n}, \cdots\right\rangle \cong Z\left(p^{\infty}\right), p c_{i 1}=0, p c_{i, n+1}=c_{i n}(n \geqq 1)$, and then setting $\alpha c_{i n}=\bar{c}_{i, n+m}$. For such an $\alpha$, there is no $\lambda: A \rightarrow A$ with $\phi \lambda=\alpha$, since $\alpha D\left[p^{m}\right] \neq 0$, but $\phi \lambda D\left[p^{m}\right]$ must vanish. This completes the proof.

In view of [3], we conclude:

COROllary. A torsion group is quasi-projective over its endomorphism ring $E$ exactly if it is a flat E-module.

The above method can be suitably generalized to describe the algebraically compact and cotorsion groups which are quasi-projective over their endomorphism rings (see a forthcoming paper by $\mathbf{A}$. Longtin).

\section{REFERENCES}

1. G. D. Poole and J. D. Reid, Abelian groups quasi-injective over their endomorphism rings, Canad. J. Math. 24 (1972), 617-621.

2. F. Richman, Detachable p-groups and quasi-injectivity (to appear).

3. F. Richman and E. A. Walker, Primary abelian groups as modules over their endomorphism rings, Math. Z. 89 (1965), 77-81. MR 32 \#2475.

4. - Modules over PID's that are injective over their endomorphism rings, Proceedings of the Park City Ring Theory Conference, Academic Press, New York (to appear).

Department of Mathematics, Tulane University, New Orleans, Louisiana 70118 